\title{
It was not accurate
}

\author{
Yang $\mathrm{Hao}^{1} \cdot$ Zhong You ${ }^{2} \cdot$ Meng-Jun $\mathrm{Wu}^{3}$
}

Received: 29 October 2019 / Accepted: 16 November 2019 / Published online: 26 November 2019

(c) Japanese Society of Anesthesiologists 2019

To the Editor:

We read with great interest the recent article by Kondo et al. [1]. Their inspirational study on this important topic deserves further discussion. In particular, we have some concerns in regards to their study conclusion; the authors have claimed the decreases in both regional cerebral blood volume (rCBV) and oxygenation ( $\mathrm{rCBO})$ after induction of spinal anesthesia for cesarean section are probably dependent on the decrease in systemic vascular resistance (SVR) due to spinal anesthesia.

It is well known that, to obtain adequate anesthesia for cesarean section (C-section), an intense blockade covering from the sacral (S2-S4) to the visceral fibers (T4-T12) is needed [2]. A blockade with such extension results in hypotension by blocking the sympathetic fibers. In the study by Kondo et al., the level of block height (mean) was Th5 (Th2-Th6) dermatome. However, an important question is raised: what was the percentages of patients with Th2-Th4 dermatome?

If the percentages of patients with Th2-Th4 dermatome were too high, the incidence of hypotension after induction

Yang Hao and Zhong You contributed equally to this study and share first authorship.

This comment refers to the article available online at https://doi. org/10.1007/s00540-019-02670-0.

Meng-Jun Wu

2014982567@qq.com

1 Department of Imaging, Nanchong Central Hospital, The Second Clinical Medical College, North Sichuan Medical College, Nanchong, Sichuan, China

2 Department of Pediatric Surgery, Chengdu Women and Children's Central Hospital, School of Medicine, University of Electronic Science and Technology, Sichuan, China

3 Department of Anesthesiology, The Affiliated Hospital, School of Medicine, UESTC Chengdu Women's and Children's Central Hospital, Chengdu, Sichuan, China of spinal anesthesia for C-section would increase. On the other hand, the high level of block height Th2 dermatome would inhibit the cardiac function, leading to decreased cardiac output [2]. Therefore, under such high incidence of hypotension and decreased cardiac function setting, spinal anesthesia rCBV and rCBO after induction of spinal anesthesia being probably dependent on the decrease in SVR might be not accurate.

From the discussion above, the current study by Kondo et al. did not provide convincing evidence that $\mathrm{rCBV}$ and rCBO after induction of spinal anesthesia for cesarean section are probably dependent on the decrease in SVR.

\section{References}

1. Kondo Y, Hirose N, Maeda T, Yoshino A, Suzuki T. Relationship between changes in regional cerebral blood volume and oxygenation and changes in cardiac output and systemic vascular resistance during spinal anesthesia in women undergoing cesarean section. J Anesth. 2019;33:579-86.

2. Ourado AD, Filho RL, Fernandes RA, Gondim MC, Nogueira EV. Sufentanil in combination with low-dose hyperbaric bupivacaine in spinal anesthesia for cesarean section: a randomized clinical trial. Braz J Anesthesiol. 2016;66:622-7.

Publisher's Note Springer Nature remains neutral with regard to jurisdictional claims in published maps and institutional affiliations. 